\title{
Building the social graph of the History of European Integration
}

\section{A pipeline for Humanist-Machine Interaction in the Digital Humanities}

Lars Wieneke ${ }^{1}$, Marten Düring ${ }^{1}$, Ghislain Silaume ${ }^{1}$, Carine Lallemand ${ }^{2}$, Vincenzo Croce $^{3}$, Marilena Lazarro ${ }^{3}$, Francesco $\mathrm{Nucci}^{3}$, Chiara Pasini ${ }^{4}$, Piero Fraternali ${ }^{4}$, Marco Tagliasacchi ${ }^{4}$, Mark Melenhorst ${ }^{5}$, Jasminko Novak ${ }^{6}$, Isabel Micheel ${ }^{6}$, Erik Harloff ${ }^{6}$, Javier Garcia Moron ${ }^{7}$

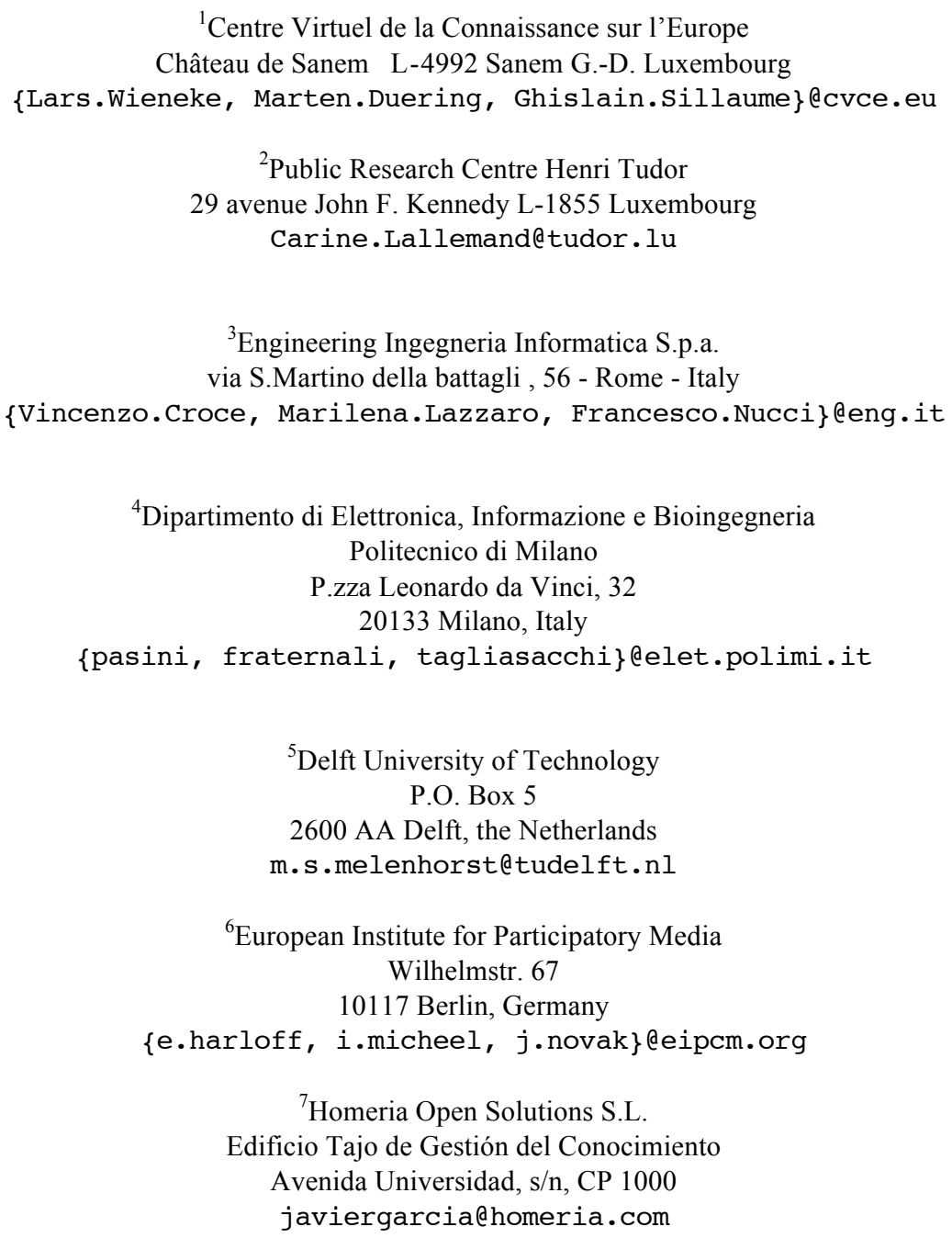




\begin{abstract}
The breadth and scale of multimedia archives provides a tremendous potential for historical research that hasn't been fully tapped up to know. In this paper we want to discuss the approach taken by the History of Europe application, a demonstrator for the integration of human and machine computation that combines the power of face recognition technology with two distinctively different crowd-sourcing approaches to compute co-occurrences of persons in historical image sets. These co-occurrences are turned into a social graph that connects persons with each other and positions them, through information about the date and location of recording, in time and space. The resulting visualization of the graph as well as analytical tools can help historians to find new impulses for research and to un-earth previously unknown relationships. As such the integration of human expertise and machine computation enables a new class of applications for the exploration of multimedia archives with significant potential for the digital humanities.
\end{abstract}

Keywords. Face recognition, Entity linking, User centered design, Data visualization, Digital Humanities, Human-machine Interaction, History, European Studies

\title{
1 Introduction
}

With the increasing digitalization of contemporary historical sources, such as images, videos or sound recordings, new opportunities for research emerge. Nevertheless, automatic processes alone are often not reliable enough to extract high-level information, such as the identity of persons that are depicted in these resources. A fully manual validation of faces and identities on the other hand creates highly reliable information but leads at the same time to a bottleneck for the indexation of larger archives. In this paper we want to discuss how the History of Europe (HoE) application overcomes these limitations through an adapted combination of human and machine computation.

Up until now the full potential of such combinations remains largely untapped due to the complexity and diverse demands of such projects: The implementation and integration of processing and recognition algorithms requires specialized know-how and users from the humanities are challenged with expressing requirements for unprecedented tasks and methods which haven't emerged yet while the final application should be useable for users who don't have a technical background. To overcome these issues HoE follows a decidedly user-centered approach that tries to fuse research in computer science, the design of human-computation tasks, data visualization, social engineering and the humanities in a coherent application.

To do the complexity of the process justice it is the goal of this paper not only to describe the current (technological) state of the HoE app and to give an outlook on its future development but also to highlight the development process itself.

The HoE app overcomes the inherent complexity of hybrid human and machine computation in part through its integration in the CUbRIK framework. CUbRIK ${ }^{1}$ is an

1 see http://www.cubrikproject.eu/ 
FP7-ICT funded 36-month long project that started in October 2011 and which focuses on building a flexible platform for multimedia search that combines human input and machine computation. The second chapter of this paper therefore gives a brief introduction into the architecture of the CUbRIK platform to provide a context for the further development of the HoE app.

In chapter three, From idea to requirements, the specification process is described as a mediation between user pull and technology push. In contrast to common software development processes which follow a specific need expressed by the final users, the initial impulse for the development of HoE emerged from the FP7 funded project CUbRIK for which HoE serves as a demonstrator application. The section will highlight how this strong technology push has been turned in an input for a group of humanities researchers during a focus group at the Centre Virtuel de la Connaissance sur 1'Europe (CVCE) in Luxembourg and how in turn the feedback of these experts was taken as an input for a feasibility review that led to the final concept for the HoE application.

Chapter four describes the different components of the HoE app in more detail. After a discussion of the image indexation pipeline and its two different approaches to crowd sourcing through the integration of both click-workers and an expert crowd, first the construction and finally the visualization of the social graph is presented.

The fifth chapter on Expert sourcing and user motivation discusses how to involve communities of experts in crowd sourcing tasks that would be too difficult to solve for non-expert users. The section starts off with a description of the general challenges for crowd sourcing, followed by the specific role of experts in the HoE app. It continues with an excurse on the nature of truth in science and the humanities. Untypical for computer science but very typical for the humanities we will discuss how the HoE app tries to enable competing interpretations of historical "facts" through the integration of multiple perspectives in the user interface rather relying only on binary opositions. The chapter concludes with a review of different kind of user motivations and derived incentives as well as their integration in the interface.

Finally the paper will give an outlook on the future development of the HoE app.

\section{From CUbRIK to the History of Europe application}

The CUbRIK approach facilitates the interaction between human input and machine computations. From the ground up CUbRIK had been designed to be a flexible platform that could be tailored to meet the specific needs of different domains. As a whole the CUBRIK platform is built for multimedia search practitioners, researchers and end-users and relies on a framework for executing processes (the so called CUbRIK pipelines), which bundle and distribute the tasks to be executed. The original concept for the CUBRIK architecture is specified in Figure 1.

Each CUbRIK pipeline is described by a workflow of tasks, allocated to executors. Task executors can be software components for data analysis, metadata indexing, search engines and the presentation of results among others. Moreover, tasks can also be allocated to human users, for example via gaming interfaces, or to an entire community, for example by a crowdsourcing tool component. 
This idea reflects the CUbRIK approach to have Humans in the loop of generic Search processes. Human activities can involve both humans playing GWAPs ${ }^{2}$ and crowd sourcing markets ${ }^{3}$ via frameworks like Amazon Mechanical Turk ${ }^{4}$ or Microtask $^{5}$.

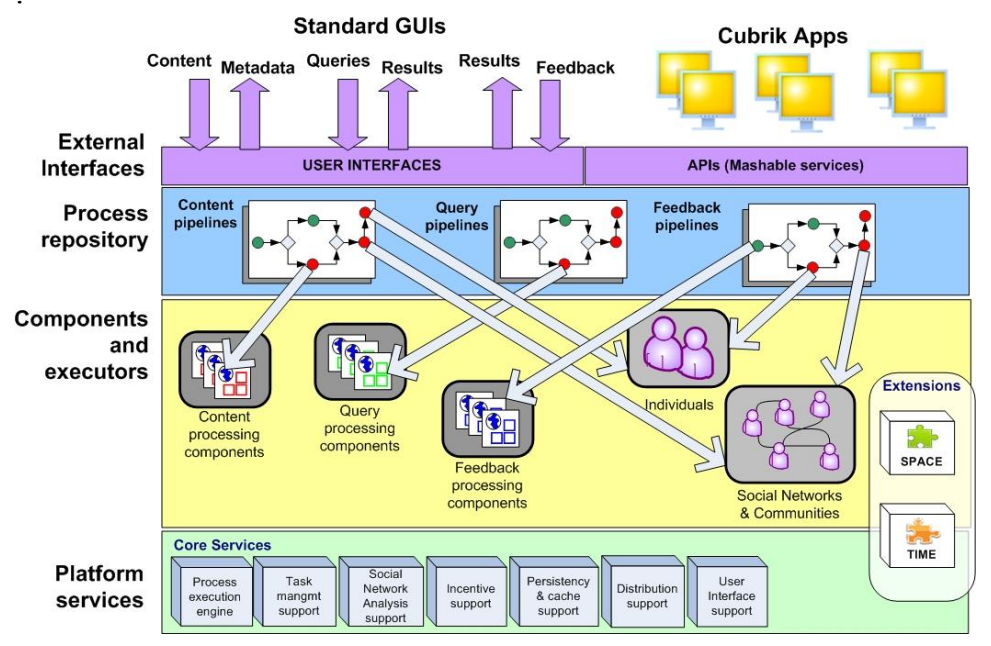

Fig. 1. Key components of the CUbRIK architecture.

\section{From idea to requirements}

The CUbRIK project is characterized through a strong interplay between technological progress and the development of applications that are tailored to the needs of the end-users. Throughout the project, efforts have been made to elicit the needs of the end-users and use their input for the requirements specification process. This can be referred to as 'user pull'.

Simultaneously, solutions have been developed that combine machine-based processing with human and social intelligence. In order to assess the added value of these solutions, an application environment is needed that allows for the technical evaluation of the solutions. This is referred to as 'technology push'.

Following a combined 'user pull' and 'technology push', approach (see Figure 2), the resulting application should then contain new technology that demonstrates the added value of combining machine-based processing with human and social intelligence, while it simultaneously should make the complex work of historians easier. The process of mapping these solutions to the needs of historians (and vice versa)

${ }^{2}$ GWAP - Games With A Purpose is the CUbRIK gamification strategy to outsource certain processes to humans while also being entertaining

${ }^{3}$ The crowd sourcing market outsources tasks to a crowd of paid workers.

${ }^{4}$ https://www.mturk.com

${ }^{5} \mathrm{http}: / /$ www.microtask.com/ 
involves an estimation of technical feasibility and alignment of different components. During this mapping process, researchers in Human Computer Interaction therefore worked closely together with multimedia information retrieval specialists.

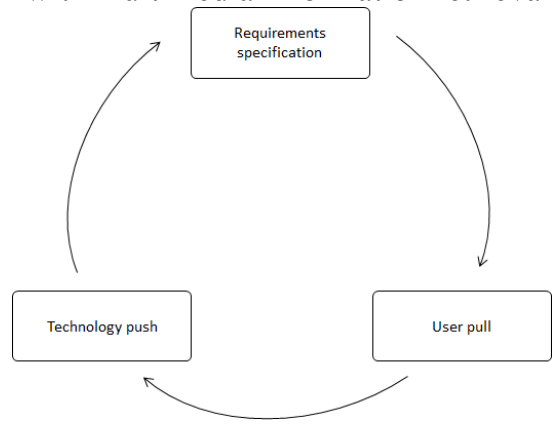

Fig. 2. The role of user pull and technology push in specifying requirements

The following steps were taken in order to combine support for the work of historians with advanced multimedia search technologies. Initially, five different user stories were developed, each describing both a task the domain experts perform as part of their daily research work, and a technological solution to support the task at hand. The user stories were evaluated with a focus group of domain experts as the future end users, an expert in the field of multimedia search and retrieval as well as CUbRIK's technical experts, the latter considering technical feasibility and relevance to the CUbRIK project.

Additionally, two exploratory interviews were conducted to get a specific idea about the work of the domain experts, its complexity, and the experts' needs for support. As a result, user stories that were both challenging from a technological perspective and promising from the point of view of the expert users were selected for further refinement.

The selection of user stories initiated the application specification process, including the design of mock-ups for each of the user stories. The mock-ups fulfilled two functions: 1) to visualize the needed features, and hence to assist the technical specification process; 2) to demonstrate what the experts would actually do with our ideas and how the application would fit into their workflow.

A second focus group with 5 CVCE-experts was set up to improve our understanding of the historians' needs, and foremost, to collect feedback on the mock-ups and the concepts behind them. The results led to modifications of the mock-ups and provided further input for the technical requirements specification process, both of which will be the basis for the CUbRIK History of Europe application.

In order to be able to evaluate whether the formulated requirements have been met, a set of success criteria has been specified, relating to both the technical performance of the pipelines that are used in the application (system oriented evaluation) as well as the extent to which the application offers added value to the history experts and how the experts evaluate the user experience (user-based evaluation). The set of success criteria will be the basis for the set up of both the system-oriented evaluation and the user-based evaluation. 


\section{The History of Europe application}

The History of Europe (HoE) application is based on a curated collection of more than 3000 images, representing the main events and actors in the history of the European integration. The collection is curated and hosted by the Centre Virtuel de la Connaissance sur l'Europe (CVCE).

In a first step, an image indexation pipeline identifies the location of individual faces in the photographs. The location of these faces is verified by a crowd of "clickworkers" with no specific training who evaluate for each recognized face if the depicted image shows a human face or not. Following the face verification process, an automatic face recognition process is triggered that associates each of the now verified faces with a list of ten possible identities. This list of candidates is then disseminated to a crowd of experts that vote and comment for their preferred identity.

Besides the identities of the different persons, all information that is associated to an image, such as the, the time or the place where the image was taken as well as contextual information about associated historical events can be reviewed by expert users and delegated to a crowd of domain experts for review. Building on the computed co-occurrence of persons in images a social graph is constructed that connects them with each other. Connections gain in strength the more often persons appear together in an image. Finally the result of this process is depicted in a visualization of the social graph with a set of analytical tools.

\subsection{Image indexation - the CUbRIK pipeline}

Images go through a pipeline that seamlessly integrates algorithms executed by a computer with human-executed tasks. Each image is scanned searching for persons, by means of a state-of-the art face detection/recognition automatic tool ${ }^{6}$. The tool consists of two basic components: the first devoted to face detection and the second to face identification. First, the face detection component receives as input a collection of images that are analyzed one by one. Once a photo is processed, the detector provides as output a collection of bounding boxes (i.e., regions of the image in which a face is detected). For each detected face the component provides: i) additional information pertaining the pose of the face; ii) the confidence score of the detection. Due to the challenging nature of the images contained in the dataset (e.g., non-frontal faces, shading, occlusions, etc.) the output of the detector contains both false positives (i.e., bounding boxes identifying regions in which no faces appear) and false negatives (i.e., some of the faces are not detected). For this reason, a crowdsourcing solution is employed to to correct for these errors, leveraging a crowd of non-expert users.

At the end of the detection phase, the face identification component extracts a biometric template from each detected bounding box. Then, a similarity score is computed between the biometric template of each unknown face and those of already known persons, which are included in an initial dataset enriched with portraits of persons whose names were known and validated by experts beforehand. When more than one

6 Kee Square, Morpheus SDK, http://www.keesquare.com 
reference template is available for a known person, the largest similarity score is retained. Then, for each unknown face, a ranked list is produced in decreasing order of similarity score. In our experiments with more than 200 known persons in the initial dataset, the top-1 element represented the correct person in $18 \%$ of the cases. In $39 \%$ of the cases the correct person was listed among the top-10 elements. Therefore, a second crowdsourcing task is performed to correctly assign the identity to each unknown face. In this case, an expert is asked to select one person in a list of 10 suggested names, or indicate another person if her/his name is not included in the suggestions.
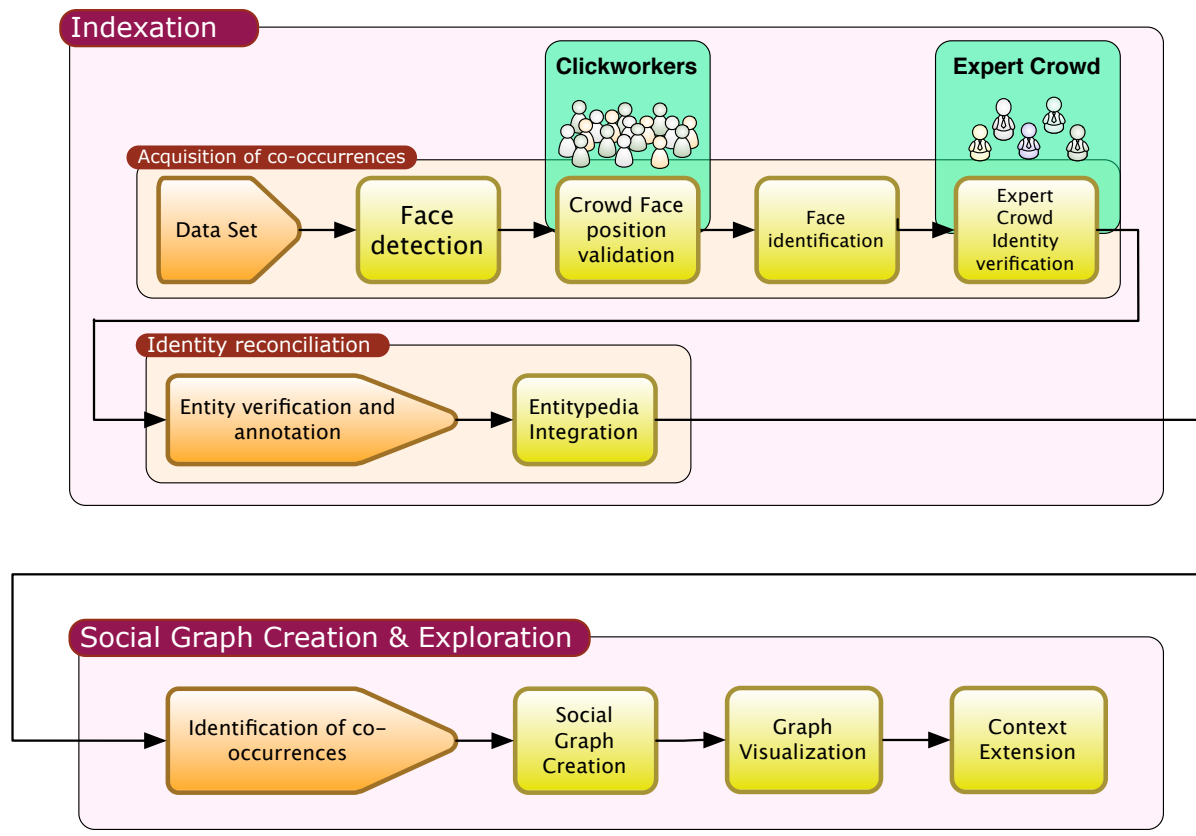

Fig. 3. High-level view of the HoE indexation pipeline

At the end of this process, an identified person is assigned to its appropriate entity in Entitypedia, a repository for linked data on historical events and people. This allows us to map a person's appearance in different images unambiguously to only one rather than many different entities with the same name. All the steps described are reiterated whenever a user enters a new image, or a new source/media type is connected to the application.

Figure 3 shows a high level view of the CUBRIK pipeline described above, which consists of a sequence of both machine-executed tasks and human-executed tasks, with the goal of increasing the quality of the result with respect to fully, automated solutions.

7 see http://entitypedia.org/ 


\subsection{The social graph}

\section{Construction of the social graph}

The basis for the construction of any graph is the availability of structurally standardized dependencies between different types of entities that are to occur in the graph. These dependencies or links often vary in meaningfulness, the so-called link weight. The social graph in the History of Europe App aims at representing and visualizing dependencies between historically relevant persons in the context of European integration. Thereby the weight of the (social) links between person entities relies on their co-occurrence in historic photographs as identified by the before mentioned image indexation process. The more frequently two persons appear in different photographs, the stronger the link between the corresponding entities in the graph (see [1] for further details).

\section{Visualization of the social graph}

The resulting social graph is visualized by means of a force-directed graph layout that groups nodes with stronger link weights closer together than nodes with weaker links in such a way that a global optimum is reached. The visualization is performed by the javascript components Crossfilter and D3.js ${ }^{8}$ which display the graph in the form of an interactive visual network of persons and connections between them. Thereby, each node represents a person and each edge represents the frequency of their co-occurrences in the given photo collection. The width of an edge represents the number of co-occurrences, in such way that a wider edge means that both persons appear simultaneously in a high number of photos (see figure 4 below).

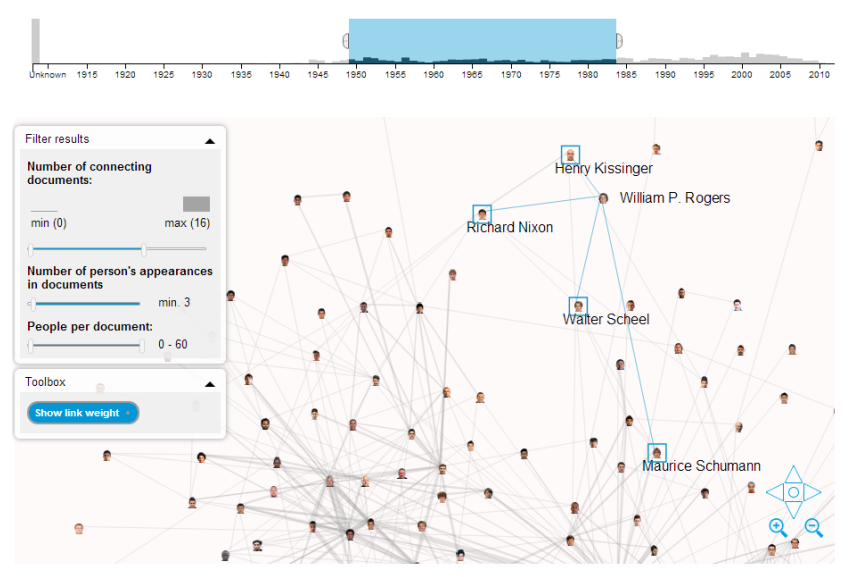

Fig. 4. Social Graph Overview

Such network visualizations have the potential to highlight unexpected connections and patterns in all kinds of relations, be they social or between other nodes such as

8 see http://d3js.org/ 
documents. In order to further evaluate any such finding it is however important to be able to interactively explore the relationships between the nodes, manipulate the parameters determining their visualization and re-contextualize the graph based on specific data subsets that are of interest to the user.

Users can interact with the History of Europe social graph in different ways, e.g. clicking on a node results on an ego-graph of the selected person and clicking on an edge displays documents that relate to both selected relationship. As the documents stored in the collection very often come with a date of creation, the graph can be filtered by date with the timeline, displaying only the connections of documents created within this timespan. This timeline also shows the amount of photos per date that are contained in the collection. Another filtering option is the number of connecting documents, which allows the visualization of those relationships that are only included in an interval of a minimum and maximum number of documents. This feature is useful to highlight highest co-occurrences. Finally, the number of appearances of a person in the processed collection lets us identify people who appear particularly often in any given time frame.

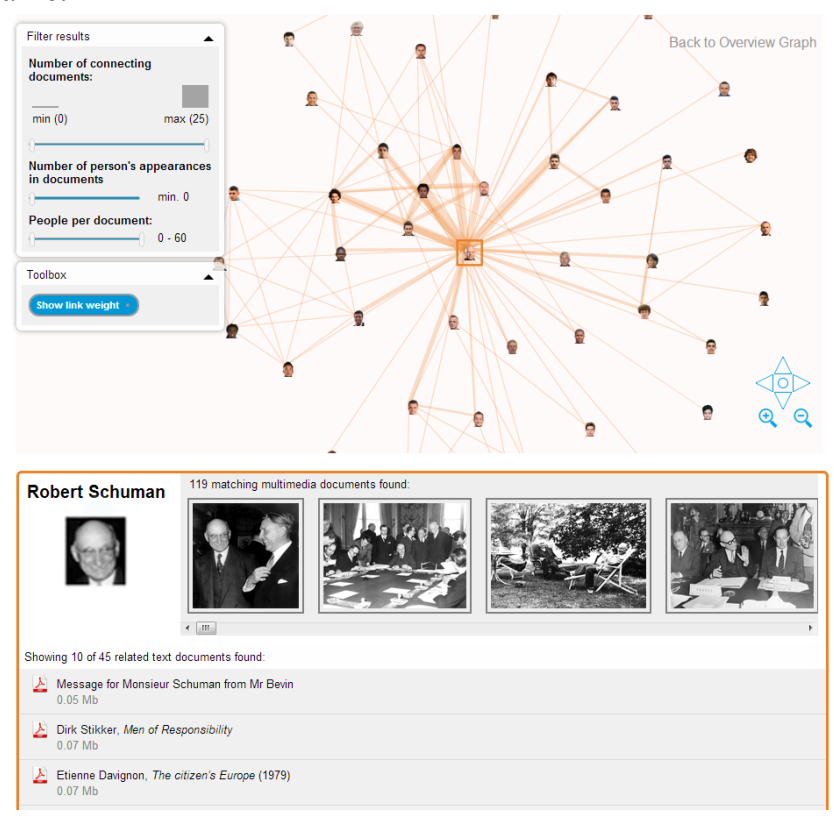

Fig. 5. Ego-Graph for Robert Schuman

Filtering of images used for the construction of the graph is also possible by defining the number of persons that were recognized in the images. This filter is mainly used to show/hide those group pictures that include a high number of people on the same picture.

After selecting a person, the graph turns into an ego-graph (see figure 5), centering on the selected person and showing all relationships for the person. All photos in which the selected person appears are displayed below the graph, sorted according to 
dates and in consideration of other filters. Users can review who is tagged in each of these photos by moving the mouse over them, which highlights the different persons that appear in the selected photo in the graph. In addition to these photos, a list of links to text documents that contain references to the selected person is displayed below the photos.

Another powerful feature is the inspection of relationships. Whenever a user clicks on an edge between two persons, the selection shows all images and documents where both persons appear in and that therefore constitute the relationship between the persons.

\subsection{Expert sourcing and user motivation}

Crowd sourcing relies on the active contribution of a large amount of persons. While existing platforms like Amazons Mechanical Turk ${ }^{9}$ provide powerful incentive mechanisms (money) to source click-workers, the same mechanisms do not necessarily apply to the demands of expert users whose specific expertise is required in the validation of person identities and other information associated to historical documents. This chapter discusses the expert sourcing tasks in detail and develops an approach based on the results of the focus groups that were conducted with expert users at the CVCE. Furthermore we outline potential motivational factors and how they can be integrated in the interface. Finally we will discuss how different perspectives on a piece of information are integrated in the interface of the app to stimulate discussions and preserve conflicting opinions beyond a binary concept of truth.

\section{From Click-workers to Expert Sourcing}

In the History of Europe application, two types of crowds are considered to solve different types of tasks driven by different types of motivation. On the one hand, click-workers are used to solve microtasks, i.e. small tasks which are easy to perform for most humans and which don't require domain knowledge. Click-workers can easily be motivated with e.g. monetary reward systems that pay workers per task. In the History of Europe application, click-workers contribute during the image indexation process by identifying and correcting false-positive and false-negative results of the face detection component via the external crowdsourcing platform "Microtask.com". However, when tasks get too complex and require more knowledge, click-workers often don't suffice. Harloff demonstrated in [2] that in the context of the History of Europe application, relying on click-workers for tasks such as the identification of persons in historic photos proves difficult: they are often of low resolution, taken from different viewpoints and depict the same persons in different historical periods. This makes face identification not only difficult for automatic methods but also for human users without appropriate background knowledge. For these kinds of tasks, a high level of domain knowledge is required which suggest the need to actively involve historians as a crowd of experts.

\footnotetext{
9 https://www.mturk.com/mturk/welcome
} 
For the design of such expert-based crowdsourcing tasks, other types of motivational models apply. In order to motivate expert users to participate in improving the results of the automatic analysis and related tasks, the History of Europe application introduces a community-based exchange model. Mechanisms of community-based question-and-answer platforms such as Stackoverflow.com are explored, where users can build up social reputation within the community the more they contribute to answering requests of other users and be ranked according to their level of expertise (see $[3,4])$. Collective action principles as they e.g. apply for Wikipedia [5], where users are also motivated by the notion of creating "public goods" by taking part in a process of collaborative knowledge building are also considered $[6,7]$.

These mechanisms strongly influenced the design of the main functionalities of the History of Europe application that aim at involving and motivating a crowd of experts. As users of the application, historians can ask specific questions, i.e. initiate new research inquiries that are associated with multimedia content such as "Who is the person on the left?" (see Figure 6). They can then rely on a community of experts with a high level of domain knowledge, both within and beyond the application, to provide answers. For this, communication channels that are already being used by the historians to exchange knowledge, e.g. e-mail and the social media service Twitter will be integrated into the History of Europe application. Thus, research inquiries can still be distributed via established channels, but in a more structured way as before, making it easier for historians to get the information they need. Furthermore the results of this exchange are fed back into the system, thus allowing an improvement of the results of the automatic methods (e.g. by back propagation of the identified faces). This is a kind of explicit expert-based crowdsourcing approach.

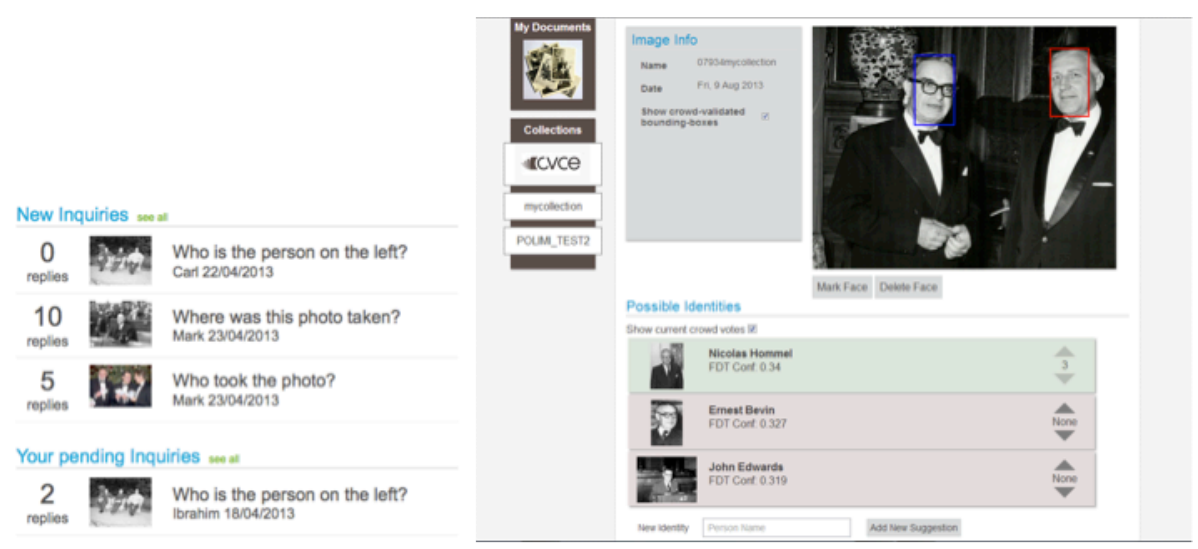

Fig.6. Research Inquiries (left) and media annotation tool considering multi-perspectivity (right) in the History of Europe application demo

At the same time, as part of their everyday research users can independently work on annotating the multimedia content of the application (see Figure 6). As the results are incorporated into the underlying knowledge base, the results of individual annotations are immediately visible and usable to others. This is an example of implicit expert- 
based crowdsourcing and collective action approach relying on the intrinsic motivation and reciprocal gratification of users.

In order for the application to be trustworthy and useful to historians in such a way that they would use it in a professional context, several challenges identified in focus groups needed to be overcome. The perceived authenticity of application by the users should be maximized, e.g. by linking users' identities from the History of Europe application to profiles from other professional networks or their official contact information. Provenance information of application content should be available and transparent. Another challenge relates to the traceability of annotations, which requires functionalities of enabling users to provide sources and explanations when making annotations. Results from automatic processes should also be as transparent as possible, not leaving any doubt of how they were calculated.

\section{Expert user motivation}

Motivational aspects were explored both from the point of view of final users (having a request) and of the Expert Crowd (participating on request of a final user in the identification or enrichment of some multimedia content). Several methods (focus group, large-scale survey, interviews and user testing) were used to explore these motivations. In the case of the History of Europe application, on the one hand, final users' primary motivations for using the application were "competence" (enhancing one's skills and general knowledge in order to feel capable and effective in one's actions) and "autonomy" (possibility of achieving tasks autonomously and having different choices to achieve one's goal). On the other hand, results showed stimulation and relatedness as the main potential motivations of the crowd. The interface had therefore to be innovative enough to be felt as interesting and exciting. Moreover, design elements able to support and highlight a relatedness experience (community aspects) were implemented within the application.

\section{Modeling opposing perspectives on truth}

Another challenge for the HoE app and the domain of the Digital Humanities in general is the conception of truth, which differs significantly e.g. to the conceptions of truth in Computer Science. Computer Scientists can rely on a stable foundation of what is true: Any experiment can be replicated and measured precisely. In the humanities the concept of truth is far more complex: It is based on the insight, that there is no neutral or objective way to study human environments. The way, in which questions are asked, how data is selected to answer them, by what means this data is analyzed and finally the way in which the results of such analyses are communicated and received all challenge the idea of "one truth". Consider the common saying "One man's terrorist is another's freedom fighter": Any such attribution inherently depends on individual viewpoints, experiences and socializations. It becomes apparent, that the interpretation and communication of historical events is directly linked to our perspective on the past as a whole. Having made this point, it is not hard to imagine how difficult it is to identify causal factors in historical events. 
The negotiation of and reasoning behind these problems are of direct relevance for the design of the History of Europe application. While there might be less conflict when it comes to associating names and faces on photographs, it is more likely that the meaning attributed to a certain photo is challenged. Just like the verbs discussed in the previous paragraph. Similarly to words, images bring with them their own "spin" on events which depend on composition, postures, gestures, angle and so on.

For these reasons, humanities scholars need the freedom to discuss competing interpretations and to see historical events and sources in the light of different research interests. In the context of the History of Europe application, scholars therefore have the opportunity to add more than one annotation, e.g. different captions to a photograph, and to comment on others, enabling academic discussion and competing interpretations. Users can vote annotations up and down, thereby relying on the wisdom of the expert crowd to identify the best annotation without simply overwriting less popular answers (see Figure 6). All these elements remain open for improvement and editing, thereby keeping the system flexible and responsive for new information.

\section{Conclusion and future work}

The History of Europe application takes on the challenge to combine cutting edge research in the domains of computer science, the design of human-computation tasks, data visualization, social engineering and the humanities by identifying synergies between the disciplines' strengths and by compensating for their weaknesses. We do this by building a pipeline which connects face recognition tools, data visualization and input from humans and creates an ongoing cycle of iteratively improved user input and machine output.

The History of Europe application stands in line with a range of other online tools for historical research ${ }^{10}$ but introduces new social features as well as crowd sourcing from both click-workers and expert users which continuously improves the system. Differently from other tools, the History of Europe application does not operate with an inflexible understanding of truth: it uses the wisdom of a crowd of experts to give input and evaluate it by relying on a robust system of discussion.

The application currently focuses on the analysis of more than 3000 photographs that chronicle the History of European Integration. In the future we will expand the selection of sources to include digitized text documents as well as audio and video interviews from different archives.

\footnotetext{
${ }^{10}$ See for example: ePistolarium (http://ckcc.huygens.knaw.nl/epistolarium ), Pelagios (http://pelagios-project.blogspot.de/), the Republic of Letters (http://republicofletters.stanford.edu/) or The Proceedings of the Old Bailey (http://www.oldbaileyonline.org/)
} 


\section{References}

1. Dioniso M, Fraternali P, Martinenghi D, Pasini C, Tagliasacchi M, Harlof E, et al. Building social graphs from images through expert-based crowdsourcing. Proceedings of the International Workshop on Social Media for Crowdsourcing and Human Computation. Paris; 2013.

2. Harloff E. Who is this person? Konzeption und prototypische Evaluierung einer Crowdsourcing-Anwendung für Multimedia-Suche. Fachhochschule Stralsund, Fachbereich Wirtschaft; 2012.

3. Preece J. Online Communities: Designing Usability and Supporting Socialbilty. 1st ed. New York, NY, USA: John Wiley \&amp; Sons, Inc.; 2000.

4. Tedjamulia SJJ, Dean DL, Olsen DR, Albrecht CC. Motivating Content Contributions to Online Communities: Toward a More Comprehensive Theory. Proceedings of the 38th Annual Hawaii International Conference on System Sciences, 2005. HICSS '05. 2005. p. 193b-193b.

5. Nov O. What motivates Wikipedians? Commun. ACM. 2007 Nov;50(11):60-4.

6. Marwell G, Oliver P. The Critical Mass in Collective Action. Cambridge University Press; 1993.

7. Novak J. Collective Action in Human Computation: From Crowd-workers to Social Collectives. Michelucci, P. (Ed.), Handbook of Human Computation. Springer; 2013. 